\title{
Nanoscale
}

CrossMark

$\leftarrow$ click for updates

Cite this: Nanoscale, 2016, 8, 4311

\section{Spectroscopic metrics allow in situ measurement of mean size and thickness of liquid-exfoliated few-layer graphene nanosheets $\dagger$}

\author{
Claudia Backes, ${ }^{a}$ Keith R. Paton, ${ }^{a}$ Damien Hanlon, ${ }^{a}$ Shengjun Yuan, ${ }^{b}$ \\ Mikhail I. Katsnelson, ${ }^{b}$ James Houston, ${ }^{a}$ Ronan J. Smith, ${ }^{a}$ David McCloskey, \\ John F. Donegan ${ }^{a}$ and Jonathan N. Coleman ${ }^{\star a}$
}

\begin{abstract}
Liquid phase exfoliation is a powerful and scalable technique to produce defect-free mono- and fewlayer graphene. However, samples are typically polydisperse and control over size and thickness is challenging. Notably, high throughput techniques to measure size and thickness are lacking. In this work, we have measured the extinction, absorption, scattering and Raman spectra for liquid phase exfoliated graphene nanosheets of various lateral sizes $(90 \leq\langle L\rangle \leq 810 \mathrm{~nm})$ and thicknesses $(2.7 \leq\langle N\rangle \leq 10.4)$. We found all spectra to show well-defined dependences on nanosheet dimensions. Measurements of extinction and absorption spectra of nanosheet dispersions showed both peak position and spectral shape to vary with nanosheet thickness in a manner consistent with theoretical calculations. This allows the development of empirical metrics to extract the mean thickness of liquid dispersed nanosheets from an extinction (or absorption) spectrum. While the scattering spectra depended on nanosheet length, poor signal to noise ratios made the resultant length metric unreliable. By analyzing Raman spectra measured on graphene nanosheet networks, we found both the D/G intensity ratio and the width of the G-band to scale with mean nanosheet length allowing us to establish quantitative relationships. In addition, we elucidate the variation of $2 D / G$ band intensities and 2D-band shape with the mean nanosheet thickness, allowing us to establish quantitative metrics for mean nanosheet thickness from Raman spectra.
\end{abstract}

Received 14th November 2015 Accepted 25th January 2016

DOI: $10.1039 / \mathrm{c} 5 \mathrm{nr} 08047 \mathrm{a}$

www.rsc.org/nanoscale form of graphene has found a wide range of applications in areas including mechanical reinforcement of composites, ${ }^{16,17}$ sensors, ${ }^{18-21}$ printable electronics, ${ }^{22,23}$ and ultrafast photonics. $^{24,25}$

For many applications, the size and thickness of few-layer graphene nanosheets is critically important. For example, counter electrodes in dye-sensitized solar cells require small nanosheets ${ }^{26}$ while mechanical reinforcement of composites requires large ones. ${ }^{16}$ While nanosheet size can be selected, often by controlled centrifugation, ${ }^{27-29}$ or informed choice of exfoliation parameters, ${ }^{13,30}$ the final size distribution needs to be measured. In addition, as production is scaled to larger volumes and becomes established as a commercial process, quality control and batch-to-batch monitoring will require continual size measurement. While statistical microscopy techniques such as atomic force microscopy (AFM) and transmission electron microscopy (TEM) can be applied to measure nanosheet size and thickness, ${ }^{13,29}$ such methods are time consuming and expensive, making them unsuitable for continual batch monitoring. Thus, there is a great need to develop fast methods for measurement of both nanosheet size and thick-

\footnotetext{
${ }^{a}$ School of Physics, CRANN and AMBER, Trinity College Dublin, Dublin 2, Ireland. E-mail: colemaj@tcd.ie

${ }^{b}$ Institute for Molecules and Materials, Radboud University of Nijmegen, Heijendaalseweg 135, 6525AJ Nijmegen, the Netherlands

$\dagger$ Electronic supplementary information (ESI) available. See DOI: 10.1039/ c5nr08047a
} 
ness of few-layer graphene produced from LPE. Such processes would facilitate lab-based characterization and are essential for commercial graphene production.

We have recently shown that for inorganic 2D materials, such as molybdenum disulfide $\left(\mathrm{MoS}_{2}\right)$, tungsten disulphide $\left(\mathrm{WS}_{2}\right)$ and gallium sulfide (GaS), optical extinction spectroscopy can be used to provide quantitative metrics for mean nanosheet length and thickness in a dispersion. ${ }^{29,31,32}$ This method allows an extremely large number of nanosheets to be sampled in a rapid measurement and has proven very useful for sample characterization. ${ }^{30}$ It has the added advantage that it uses equipment that is cheap and widely available.

However, compared to $\mathrm{MoS}_{2}, \mathrm{WS}_{2}$ and GaS, graphene is a much more common and widely studied material for which no rapid size characterization technique exists. What is urgently needed is a spectroscopic method to measure the mean size and thickness of liquid-exfoliated, few-layer graphene nanosheets in a manner similar to the methods recently reported for $\mathrm{MoS}_{2}, \mathrm{WS}_{2}$ and GaS. In this work, we develop spectroscopic techniques to analyze size-selected graphene dispersions to yield mean nanosheet size and thickness as well as improved accuracy for concentration measurements. We show that optical extinction spectroscopy can provide quantitative metrics for nanosheet thickness, while Raman spectroscopy is used to provide metrics for both nanosheet length and thickness. The metrics are reasonably robust towards different stabilizers and also hold for stock-like dispersions. Both these approaches offer a significant cost and time saving over current methods for production-scale quality control and process development.

\section{Results and discussion}

\section{Size selection}

To establish quantitative spectroscopic metrics for both mean lateral nanosheet size, $\langle L\rangle$, and thickness (expressed as number of monolayers per nanosheet), $\langle N\rangle$, the dependence of the optical response on $\langle L\rangle$ and $\langle N\rangle$ needs to be determined. To achieve this, it is first necessary to prepare a set of graphene dispersions with controlled nanosheet size and thickness. We used LPE to prepare stock dispersions from two different graphite types, obtained from Sigma Aldrich and Asbury Carbons from shear-exfoliation in aqueous sodium cholate solution (in addition, a number of other dispersion types were studied for comparison, see Methods and ESI $\dagger$ ). These were then separated into fractions with varying nanosheet sizes and thicknesses by liquid cascade centrifugation, ${ }^{32}$ a technique which uses a sequence of centrifugation steps to produce fractions with different nanosheet sizes and thicknesses. For each fraction, the length distributions of the graphene sheets were measured using statistical analysis by transmission electron microscopy (TEM, see Methods and ESI Fig. S1-S5†). Representative TEM images and some of the derived nanosheet length histograms are presented in Fig. 1A-C (see also ESI Fig. $\mathrm{S} 1 \dagger)$. The average nanosheet lengths, i.e. the arithmetic number average (or number mean) $\langle L\rangle$, varied in the range $90 \leq\langle L\rangle \leq 810 \mathrm{~nm}$ across the size-selected dispersions.

While measurement of nanosheet lengths by TEM is reasonably straightforward, measurement of nanosheet thickness is carried out by atomic force microscopy (AFM, ESI section $1.2 \dagger$ ) which is more challenging in the case of LPE nanosheets. For solution processed samples, AFM generally measures an apparent height rather than the true height due to the presence of adsorbed solvent or surfactant as well as tipsurface effects. ${ }^{13,29}$ In addition, the nanosheets can aggregate on deposition giving spurious thickness data. To minimize aggregation, the samples were dropcast on pre-heated $\mathrm{Si} / \mathrm{SiO}_{2}$ wafers $\left(120{ }^{\circ} \mathrm{C}\right)$ to accelerate drying. Aggregates were not included in the statistical analysis. To test for the effect of aggregation on the AFM statistics, mean nanosheet length as measured by AFM was plotted versus mean length from TEM in Fig. 1D. We find a good correlation confirming the consistency of both AFM and TEM, with length being slightly overestimated from AFM in the case of small nanosheets due to tip broadening and pixilation effects. To convert the measured, apparent thickness of the nanosheets to the number of layers, we applied step height analysis as described previously. ${ }^{13,29,32,33}$ The resultant histograms of number of layers per nanosheet and representative AFM images are shown in Fig. 1E-G, Fig. S2 and S3.† These data show variations in mean number of layers (arithmetic number average) $\langle N\rangle$ in the range $2.7 \leq\langle N\rangle \leq 10.4$.

We note that centrifugation-based size-selection typically separates small and thin nanosheets from larger, thicker ones. This is also because liquid exfoliation often produces smaller nanosheets that tend to be thinner and larger nanosheets that tend to be thicker. ${ }^{29,34}$ Such a correlation can be best observed when plotting the number of layers as a function of nanosheet area (estimated as measured length $\times$ width from AFM) as shown in Fig. 1H. Compared to other systems that showed a very well defined length-thickness relationship (e.g. $\mathrm{MoO}_{3}$, $\mathrm{MoS}_{2}$ or $\mathrm{GaS}^{29,31,34}$ produced by sonication), the correlation is hardly apparent in the case of the shear-exfoliated graphene under study here. In addition to the samples described above, we have also analyzed a stock-like dispersion (supernatant after mild centrifugation to remove non-exfoliated graphite, ESI section $1.3 \dagger$ ) and varied both stabilizer and exfoliation method to some extent (see Methods and ESI section 1.4†). These yield samples with a different $\mathrm{N}-\mathrm{L}$ relationship (Fig. S12 $\dagger$ ) and different chemical environments to test the robustness of the metric described below.

\section{Extinction/absorbance/scattering spectroscopy}

Extinction spectroscopy is commonly used to determine the dispersed concentration of liquid-exfoliated graphene (see below). However, in the case of other 2D materials, it has been shown that the optical spectra also contain information on both nanosheet size and thickness. ${ }^{29,31}$ This has led to the development of spectroscopic metrics that allow mean nanosheet size and thickness to be extracted from optical spectra for $\mathrm{MoS}_{2}$ and $\mathrm{GaS}^{29,31}$ If this was the case for gra- 

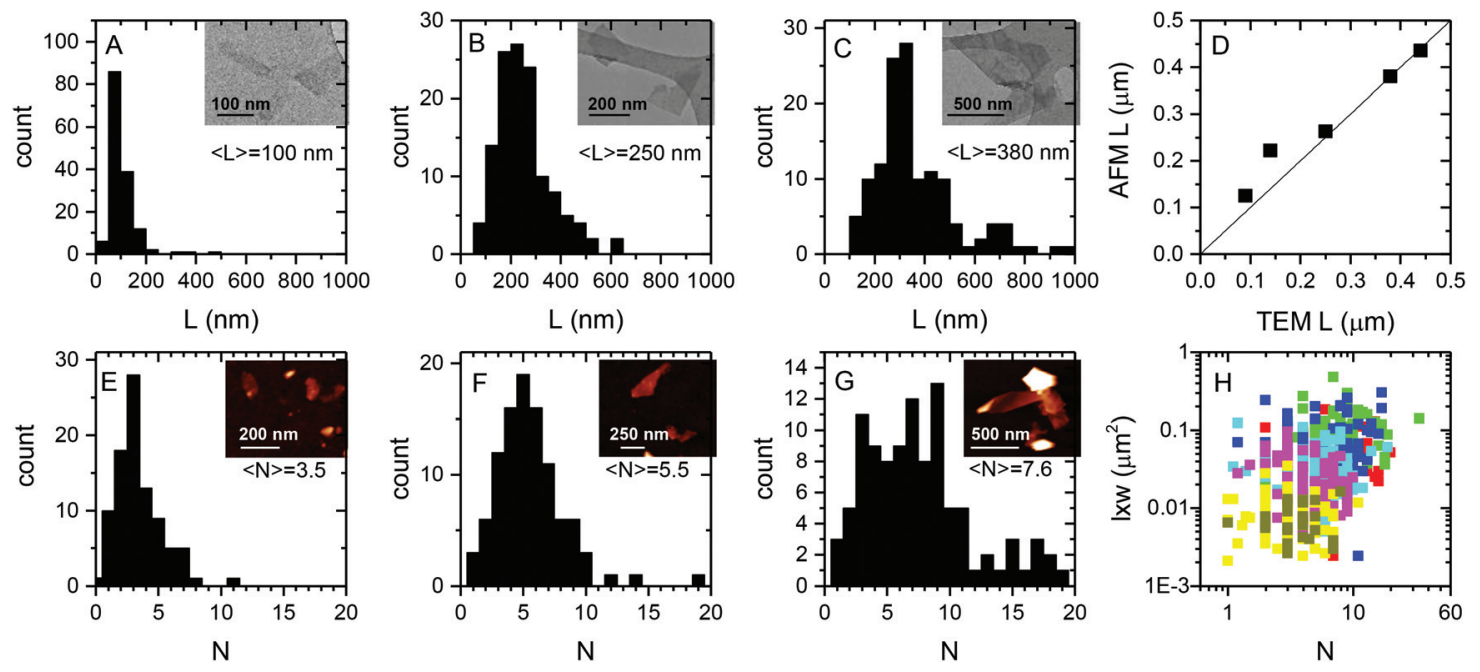

Fig. 1 Characterization of size-selected graphene dispersions by microscopy. (A)-(C) Nanosheet length was measured using TEM (representative images see inset), giving histograms from which the average nanosheet size can be determined. The data shown is a subset of the samples analyzed. Additional data see ESI. $\dagger$ (D) Plot of nanosheet length measured from AFM and TEM showing good correlation between the two microscopy techniques. (E)-(G) AFM number of layer histograms for a subset of samples (additional data see ESI $\dagger$ ). The insets show representative images $(\mathrm{H})$ a weak correlation was observed between the area of nanosheets and the thickness, in both cases measured by AFM. Colors denote nanosheets measured from different dispersions. We note that this correlation is less pronounced than for inorganic layered materials studied previously (e.g. MoS ${ }_{2}$ ).

phene, it would be an extremely powerful tool for measurement and control of sizes/thicknesses which will be important for many applications.

To identify which properties of the optical spectra might be dependent on nanosheet dimensions, we began by using the tight-binding propagation method ${ }^{35,36}$ to simulate the optical absorbance spectra for few-layer graphene with different number, $N$, of layers from 1 to 6 (Fig. $2 \mathrm{~A}$ and Methods). We found distinct changes to the spectral shape as a function of layer number. In particular, the ratio of intensity of the $\pi-\pi^{*}$ peak $(\sim 265 \mathrm{~nm})$ to the long wavelength plateau $(\sim 550-800 \mathrm{~nm})$ increases with increasing nanosheet thickness. In addition, the position of the peak also shifts to higher wavelengths with increasing nanosheet thickness. Interestingly, the changes to the optical response seem to be largely independent of the stacking sequence of the individual graphene layers in the fewlayer graphene (Fig. S13†) so that they can potentially be used as metrics to extract layer number from the optical spectra.

However, in practice, one problem associated with attempting to measure the absorbance of liquid-exfoliated graphene is that, in general, almost all experiments actually measure the extinction, Ext, of the dispersed graphene. This quantity is related to the transmission via $T=10^{-\mathrm{Ext}}$ and is what the vast majority of researchers mean when they quote the "absorbance" of nano-particulate dispersions. The reason for the discrepancy is that dispersed nano-objects tend to scatter light strongly so that both absorbance and scattering affect the measured transmittance leading to the measurement of extinction rather than absorbance..$^{29,37}$

In general, the extinction is just the sum of absorbance (Abs) and scattering (Sca) such that Ext $=$ Abs + Sca. The measured extinction can be converted to an extinction coeffi- cient, $\varepsilon$, using the Beer-Lambert law, Ext $=\varepsilon C l$, where $C$ is the nanoparticle (here nanosheet) concentration and $l$ is the cell length. This means the extinction coefficient is just the sum of absorbance $(\alpha)$ and scattering $(\sigma)$ coefficients: $\varepsilon(\lambda)=\alpha(\lambda)+$ $\sigma(\lambda) .{ }^{16}$ Note that both $\alpha$ and $\sigma$, and so $\varepsilon$, may be size-dependent as well as wavelength-dependent. This is of great importance, in particular when attempting to establish spectroscopic metrics. We and others have recently shown ${ }^{29,37}$ that the true absorbance spectrum can be measured by acquiring the spectrum inside an integrating sphere where all scattered light is collected. With knowledge of the extinction spectrum, the scattering spectrum of a given dispersion can then be determined. We have performed these measurements on the size-selected graphene dispersions under study here. In all cases, after optical characterization, the dispersions were filtered through small-pore-size filters and the graphene mass determined by careful weighing after extensive washing with water to remove most of the surfactant. The mass of residual surfactant was then determined by thermogravimetric analysis, giving the true graphene mass (see Methods and ESI, Fig. S14†). This allows the as-measured extinction, absorbance and scattering spectra to be transformed into extinction, absorbance and scattering coefficient spectra.

These are shown for each of the size-selected graphene dispersions in Fig. 2B-D, Fig. S15 and S16. $\dagger$ It is clear that they all exhibit well-defined spectral changes as a function of size. Importantly, both measured absorbance and extinction spectra are qualitatively similar to the simulated absorbance spectra, suggesting that number of layers can indeed be determined optically. We note that the measured spectra are broader and less well-defined compared to the theoretical spectra, presumably because, in spite of the size-selection, the 

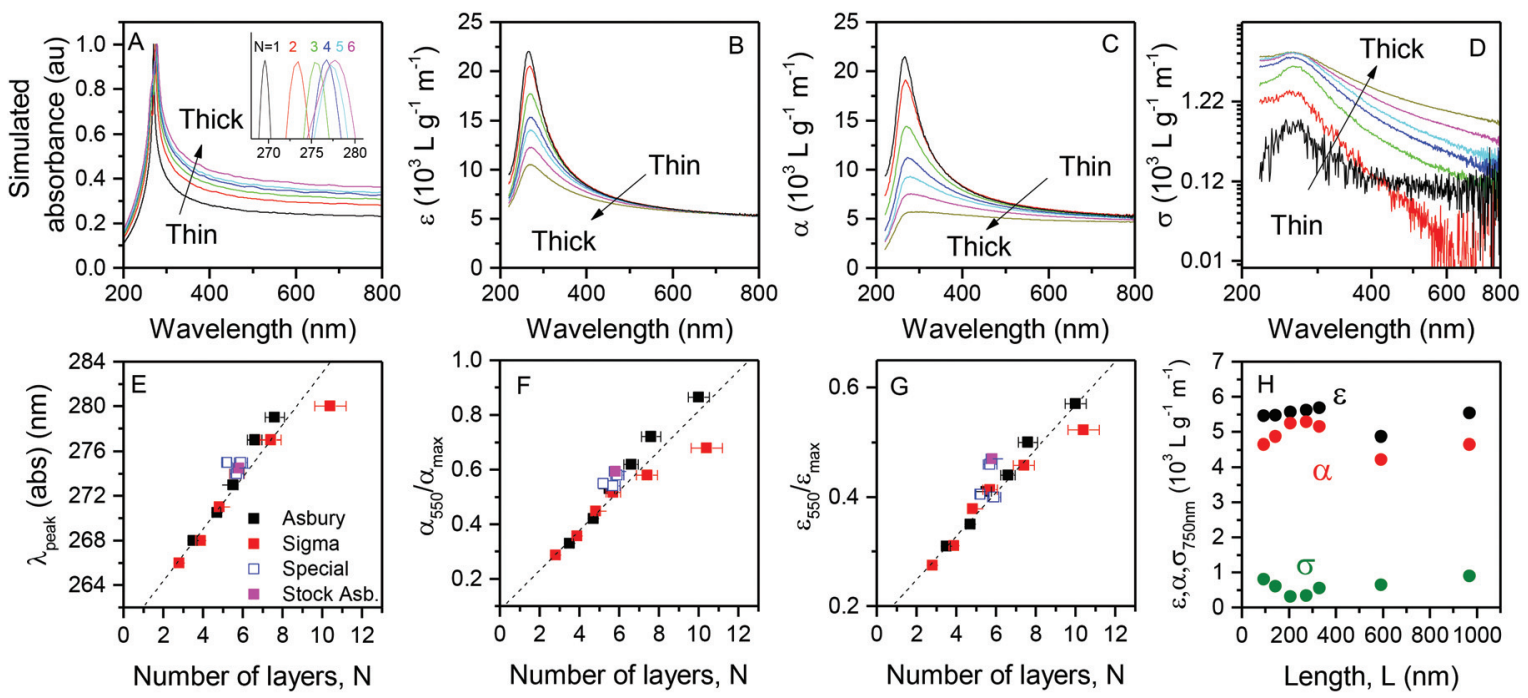

Fig. 2 UV-vis spectroscopic characterization of the size-selected dispersions. (A) Simulations of the absorbance spectra normalized to the maximum for graphene with varying thickness showing clear changes in peak intensity and position. Inset: enlarged view of the peak maximum. Thicknesses are given in the inset. (B) Measurement of the extinction spectra of our size-selected dispersions exhibiting similar changes with nanosheet size/thickness (Asbury graphite). By separating the absorbance spectra (C) from the scattering spectra (D) it is clear that the extinction is dominated by absorbance processes. (E) Position of $\pi-\pi^{*}$ absorbance peak as a function of average number of monolayers per nanosheet in the dispersion. (F) Absorbance intensity at $550 \mathrm{~nm}$ normalized to the absorbance at the peak maximum, plotted as a function of layer number. (G) Extinction intensity at $550 \mathrm{~nm}$ normalized to the extinction at the peak maximum, plotted as a function of layer number. Dotted lines in panels $\mathrm{E}-\mathrm{F}$ are empirical fits to determine $N$ according to eqn (1)-(3). Full datasets are shown for size-selected, shear-exfoliated Asbury (black) and Sigma-Aldrich (red) graphites in $\mathrm{H}_{2} \mathrm{O}$-SC. The open blue symbols correspond to data from Sigma-Aldrich graphite exfoliated in a kitchen blender with fairy liquid as stabilizer, bath sonicated Timrex graphite in $\mathrm{H}_{2} \mathrm{O}-\mathrm{SC}$ and shear-exfoliated Qingdao graphite in $\mathrm{H}_{2} \mathrm{O}-\mathrm{SC}$. The magenta symbol corresponds to a stock dispersion of Asbury graphite shear-exfoliated in $\mathrm{H}_{2} \mathrm{O}-\mathrm{SC}$. (H) Beer-Lambert coefficients, measured at 750 nm, as a function of nanosheet length. This confirms that extinction spectroscopy is a valid approach to measure concentration of dispersed nanosheets, even when nanosheet size varies considerably as long as the high wavelength region is used.

dispersions are still somewhat polydisperse containing a distribution of nanosheet sizes and thicknesses. Interestingly we find the experimental absorbance in the high wavelength region to be independent of nanosheet dimensions. This is consistent with Nair's model, ${ }^{38}$ which implies that the total light absorbed in this region depends only on the mass of graphene present. However, both the intensity and position of the $\pi-\pi^{*}$ peak $(\sim 270 \mathrm{~nm})$ appear very sensitive to the nanosheet dimensions.

To express the spectral changes, we first plot the position of the $\pi-\pi^{*}$ peak as measured from the absorbance spectra as a function of mean number of layers determined from AFM for the size-selected graphene dispersions (Fig. 2E). As suggested by theory, the peak red-shifts with thickness for both graphite sources, with a shift of $\sim 13 \mathrm{~nm}$ for a change from $\sim 3$ layers to $\sim 7$ layers. The data from the significantly more polydisperse stock-like dispersion (magenta square) falls on the same curve confirming that size-selection is not required for the metric to be applicable. Interestingly, the data points from alternative exfoliation methods such as bath sonication and shear exfoliation in a kitchen blender fall on the same curve (open blue symbols), even though in the case of the sample produced in the blender, fairy liquid, a common household kitchen soap, was used as stabilizer. This suggests that the spectral changes are quite robust towards the chemical environment. This is surprising, as solvatochromic shifts of the peak may be expected when the environment around the graphene is varied. However, these effects may not be as severe as expected in the case of the few-layer graphene under study here, with the spectral profile being governed by graphene-graphene interactions as opposed to graphene-solvent or graphenesurfactant interactions that would dominate in the case of monolayered graphene.

As a result, we suggest that the peak position can be used as quantitative metric to determine the mean number of monolayers per nanosheet in a given dispersion. By fitting an empirical function to the data, we find that the mean nanosheet thickness, $\langle N\rangle$, can be determined using eqn (1):

$$
\langle N\rangle=0.42 \lambda_{\text {peak }}(\mathrm{nm})-108
$$

where the peak position, $\lambda_{\text {peak }}$, is measured from the absorbance spectrum. We have performed similar analysis for data derived from the extinction spectra (see ESI, Fig. S17†). While the extinction measurement is more practical, we find the quantitative correlation for nanosheet thickness to break down for $N>6$, due to the contribution from scattering. Unfortunately, this greatly limits its applicability.

However, one problem with using the peak position as a metric is that the experimentally observed peak is rather broad, making precise determination of the peak position challenging. In addition, we cannot completely rule out that solvatochromic effects may shift the peak position in different 
environments, especially in the regime of very small $N$, where more and more graphene mass is in contact with the environment as the nanosheets get thinner. This may limit the usefulness of this metric in environments which vary considerably from those studied here.

To avoid this problem we can use an alternative metric. In Fig. $2 \mathrm{~F}$ we plot the intensity ratio of the absorbance at the $\pi-\pi^{*}$ peak (local maximum, $\sim 275 \mathrm{~nm}$ ) to the high wavelength plateau (for example at $550 \mathrm{~nm}$ ) as a function of mean number of layers of the graphene in the dispersion. Again, we find a well-defined relationship, with all data (including the stocklike dispersion and samples produced from alternative exfoliation or with different stabilizers) collapsing on the same curve. Again, empirical fitting shows $\langle N\rangle$ to be given by:

$$
\langle N\rangle=13.7 \times \alpha_{550} / \alpha_{\max }-1.2
$$

As previously observed, the extinction spectrum roughly follows the absorbance spectrum in shape. Hence, information encoded in the absorbance spectra will likely be extractable from the extinction spectra. This is of course more practical because it does not require an integrating sphere and can be measured in any conventional UV-vis-nIR spectrometer. To test this, we use the data in Fig. 2B to plot the ratio of extinction at the $\pi-\pi^{*}$ peak to that at the high wavelength plateau (also taken at $550 \mathrm{~nm}$ ) versus $\langle N\rangle$ in Fig. 2G. We find a relation that is very similar to the absorbance spectra again with all data collapsing on the same curve:

$$
\langle N\rangle=25 \times \varepsilon_{550} / \varepsilon_{\max }-4.2
$$

From the scatter in the data used to generate this metric we estimate the relative error in the extracted values of $\langle N\rangle$ to be $15 \%$. We note that both absorbance and extinction can be used to estimate $\langle N\rangle$ not only because graphene has a high absorption coefficient so that the extinction spectrum is dominated by absorbance rather than scattering, but also because scattering follows the absorbance in shape (see below).

However, one problem with the intensity ratio, $\varepsilon_{550} / \varepsilon_{\max }$, is that many solvents and surfactants, for example the common solvent $N$-methyl-2-pyrrolidone, absorb light in the UV region. This will mask the peak, making this metric unusable in many cases. To address this, we show in the ESI (Fig. S18†) that intensity ratios measured at different wavelengths (e.g. $\varepsilon_{550} /$ $\varepsilon_{325}$ ) can be used equally well as metrics to extract $\langle N\rangle$ from extinction spectra. Fitting this data gives the metric

$$
\langle N\rangle=35.7 \times \varepsilon_{550} / \varepsilon_{325}-14.8
$$

From the scatter in the data used to generate this metric we estimate the relative error in the extracted values of $\langle N\rangle$ to be $\sim 20 \%$. We note that we have included a data point in Fig. $\mathrm{S} 18 \dagger$ from graphene exfoliated in NMP, finding it to fall on the same curve as the samples exfoliated in different surfactants. This further confirms the robustness of this metric toward environmental changes.

Previous work on semiconducting nanosheet dispersions has shown the scattering spectrum in the high-wavelength, non-resonant regime to scale as $\sigma \propto \lambda^{-n}$, where $n$ is the scattering exponent. ${ }^{11,39}$ In addition, we recently found that for both $\mathrm{MoS}_{2}$ and GaS dispersions, the scattering exponent, $n$, scales with the lateral size of the dispersed nanosheets. ${ }^{29,31,39}$ The measured scattering coefficient spectra for graphene are shown in Fig. 2D. The scattering coefficients are generally much smaller than the absorbance coefficients, especially at high wavelength as would be expected for such a highly absorbing material. ${ }^{38}$ The spectra display a weak peak close to $270 \mathrm{~nm}$ followed by a power law decay. The latter effect is unexpected as we have previously only observed such power laws in scattering spectra in the non-resonant regime. Measuring the exponent, $n$, in the wavelength range $600-800 \mathrm{~nm}$ and plotting versus mean nanosheet length shows a well-defined relationship between $n$ and $\langle L\rangle$, similar to that observed for other materials (Fig. S19†). ${ }^{29,31,39}$ Unfortunately, in the case of graphene, the high absorption coefficient and relatively low contribution from scattering introduces a large error in the determination of the scattering exponent due to noise, such that the relationship is not as reliable as it is for other materials. However, we nevertheless analyzed the extinction spectra in a similar way offering a reasonably good metric for determination of lateral dimensions as discussed in the ESI and shown in Fig. S19. $\dagger$

To date, the most common use of UV-vis spectroscopy of graphene dispersions has been to quantify the concentration of dispersed graphene via the extinction coefficient (although this is usually erroneously referred to as the absorption coefficient). However, different values of graphene extinction coefficients have been reported for different stabilizing dispersants. $^{8,40-42}$ This may be because, as we have shown above, the optical spectra are clearly dependent on nanosheet size and thickness, especially at low wavelength. This means that the extinction coefficient is not necessarily independent of nanosheet size at all wavelengths, resulting in variation in the published values. In addition, residual solvent or surfactant adsorbed onto the graphene during the mass measurement may not always have been accounted for, leading to a large spread in reported values. Here we address this. As can be seen from the coefficient spectra in Fig. 2B-D, the coefficient at the high wavelength plateau is relatively independent of size/thickness. This suggests the high-wavelength plateau can be reliably used to assess the concentration of liquid-exfoliated graphene (Fig. 2H) across all nanosheet sizes produced under normal circumstances. The size-independent extinction coefficient at $750 \mathrm{~nm}$ was determined as $\varepsilon_{750}=5450 \mathrm{~L} \mathrm{~g}^{-1} \mathrm{~m}^{-1}$ and is reasonably consistent with values previously reported from solvent and surfactant dispersions ${ }^{40,41}$ and close to the value of $4890 \mathrm{~L} \mathrm{~g}^{-1} \mathrm{~m}^{-1}$ reported recently by Texter et al. $(500 \mathrm{~nm}) .{ }^{43} \mathrm{We}$ note that the absorption coefficient is also relatively size independent with a mean value of $\alpha_{750}=4861 \mathrm{~L} \mathrm{~g}^{-1} \mathrm{~m}^{-1}$. It is worth noting that this value is within $15 \%$ of the theoretical value of $\alpha=4237 \mathrm{~L} \mathrm{~g}^{-1} \mathrm{~m}^{-1}$, which was calculated for graphene dispersions from the intrinsic monolayer absorption. ${ }^{44}$ Additional plots of extinction, absorbance and scattering coefficients at different spectral positions are presented in the ESI, Fig. S20. $\dagger$ 


\section{Raman spectroscopy}

Beyond extinction spectroscopy, Raman spectroscopy is perhaps one of the most common characterization methods for graphene, yielding information on many properties, including defect density, doping level, number of layers, functionalization and strain. ${ }^{45-48}$ For example, it is well established that the $I_{\mathrm{D}} / I_{\mathrm{G}}$ ratio changes with nanosheet size, as in the absence of basal-plane defects, nanosheet edges are required to activate the D-band. ${ }^{49-52}$ Similarly, the shape and intensity of the 2Dband $\left(\sim 2750 \mathrm{~cm}^{-1}\right)$ changes with number of layers, ${ }^{45,53,54}$ with monolayer graphene giving a single narrow peak with roughly twice the intensity of the G-band. For many of these parameters however, quantitative measurements have only been carried out on single nanosheets of micromechanicallycleaved graphene, ${ }^{46,53}$ which has limited application to bulkproduced LPE-samples. In the case of LPE samples, typically only qualitative analysis of the Raman spectra is performed $^{27,55-58}$ that does not allow for nanosheet length (in $\mathrm{nm}$ ) or thickness (i.e. mean number of layers) to be extracted from the spectrum.

In order to derive quantitative metrics for the characterization of ensembles of nanosheets, we have produced films of re-aggregated graphene by vacuum filtration through alumina membranes. We note that this is entirely different from probing individual nanosheets deposited on a substrate, as we probe thousands of nanosheets simultaneously in a single measurement (also see ESI section 3.3†). We therefore expect similar trends in the Raman spectra as function of size and thickness as for micromechanically-cleaved single sheets, but with a different quantification. Our films were prepared from dispersions that were size-selected by centrifugation, as described above. Such films are typically hundreds of nanometers thick and consist of porous ( $\sim 50 \%$ free volume), disordered networks of nanosheets with, on average, $\sim 25000$ nanosheets per $\mu \mathrm{m}^{3} \quad\left(800-85000 \mu \mathrm{m}^{-3}\right.$ depending on nanosheet size). The Raman spectra (532 nm excitation wavelength) were collected with a spot size of $\sim 1 \mu \mathrm{m}$. Assuming a penetration depth of $\sim 250 \mathrm{~nm},{ }^{59}$ this means that the beam interrogates a minimum of 200 nanosheets per measurement (assuming the film thickness is not much smaller than $200 \mathrm{~nm}$ ). Averaging over 120 measurements from different spatial positions effectively means we are collecting data from a minimum of 25000 nanosheets per sample. This is in marked contrast to measurements on individual sheets in particular because it means we will always probe edges of nanosheets and have effects from orientation, strain, doping etc. However, these effects will average out within each sample due to the large number of nanosheets sampled so that samples of LPE graphene can be compared to other samples of LPE graphene even though they do not necessarily quantitatively compare to other graphene (or nanographite) samples.

Fig. 3A shows the D-, G- and $\mathrm{D}^{\prime}$-peaks, with the intensity normalized to the G-peak of the Raman spectra of the LPE samples as described above. Clear changes can be seen in all bands as the nanosheet size is varied. As expected, the inten-
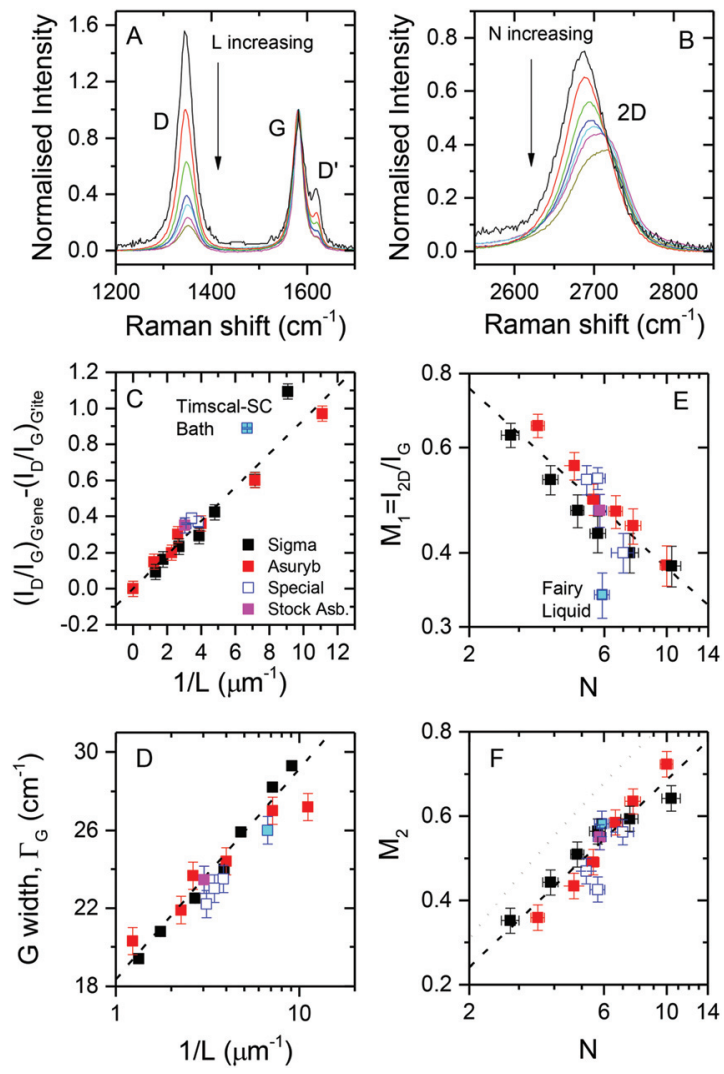

Fig. 3 Characterization of size-selected dispersions by Raman spectroscopy on filtered films of restacked graphene. (A)-(B) Raman spectra $\left(\lambda_{\text {exc }}=532 \mathrm{~nm}\right.$, Asbury graphite) showing significant changes in both the $D$-band (A) and 2D-band (B) when normalized to the G-band intensity. As the nanosheet size is decreased, the D-band intensity increases, while the 2D-band becomes narrower with a higher peak intensity. NB The color scheme used in A and B is the same as that used in Fig. 2B-D. (C) After subtracting the effect of defects present in the parent graphite material, the $I_{\mathrm{D}} / I_{\mathrm{G}}$ ratio shows the expected linear dependence on the inverse of the nanosheet size. The dashed line is a fit to eqn 4. (D) Plotting the FWHM of the G-band as a function of nanosheet length, offers an alternative metric for this parameter according to eqn (5). ${ }^{13}$ (E) Plot of the intensity of the 2D-band at its maximum of the normalized spectra $I_{2 \mathrm{D}} / I_{\mathrm{G}}$ (defined as $M_{1}$ ) as a function of mean layer number. The dashed line is a fit to eqn (6). (F) Plot of metric $M_{2}$ to quantitatively relate changes in the 2D-band to the mean number of layers, $\langle N\rangle$. The dashed line is a fit to eqn (8). The dotted line in $F$ shows the relationship between $M_{2}$ and $N$ found for mechanically cleaved graphene. (C-F) Full datasets are shown for size-selected, shear-exfoliated Asbury (black) and Sigma-Aldrich (red) graphites in $\mathrm{H}_{2} \mathrm{O}-\mathrm{SC}$. The open blue symbols correspond to data from Sigma-Aldrich graphite exfoliated in a kitchen blender with fairy liquid as stabilizer, bath sonicated Timrex graphite in $\mathrm{H}_{2} \mathrm{O}$-SC and shear-exfoliated Qingdao graphite in $\mathrm{H}_{2} \mathrm{O}-\mathrm{SC}$ as well as NMP. The magenta symbol corresponds to a stock dispersion of Asbury graphite shear-exfoliated in $\mathrm{H}_{2} \mathrm{O}-\mathrm{SC}$.

sity of the D-band increases as the nanosheet size falls, as does the $\mathrm{D}^{\prime}$ band intensity. The shape and intensity of the $2 \mathrm{D}$ band also shows clear changes with nanosheet size (Fig. 3B), with the peak intensity increasing and the width narrowing as the size decreases. The position of the maximum of the 2D-band also shifts to lower wavenumber with decreasing nanosheet size. We propose that a number of size-metrics can 
be derived from these spectral changes. Again, we analyze two full sets of size-selected LPE graphene from two different graphite sources, as well as a stock-like dispersion and samples produced from different exfoliation techniques and with different stabilizers such as sodium cholate, fairy liquid and NMP. Since residual solvent and surfactant cannot be completely removed by washing the filtered films with water (see Fig. S14 $\dagger$ ), it is important to test whether this has an impact on the Raman spectra due to potential doping. ${ }^{60-62}$ In the ESI, $\dagger$ we also show Raman spectra of the stock-like dispersion obtained from restacked films after filtration (our standard methodology) compared to spectra acquired on a dried droplet (with no surfactant removed by washing) and in liquid (Fig. S7†). The mean spectrum measured on the dried droplet is identical to the spectrum obtained from the filtered film suggesting environmental effects from surfactant to be negligible. The measurement in liquid yielded a slightly different spectrum presumably due to orientation effects (see ESI $\dagger$ ) suggesting that the quantitative relations derived below are only applicable to restacked films and would require a different calibration for liquid dispersions (with the exception of the G-width length metric, see below).

The simplest metric, which has previously been used to qualitatively describe the average nanosheet size of LPE samples, ${ }^{27}$ is the $I_{\mathrm{D}} / I_{\mathrm{G}}$ ratio. We have shown in the ESI (section $\mathrm{S} 3.3 \dagger$ ) that the $\mathrm{D} / \mathrm{G}$ ratio measured for an ensemble of graphene nanosheets, $\left(I_{\mathrm{D}} / I_{\mathrm{G}}\right)_{\mathrm{G}^{\prime} \text { ene }}$, is given by $\left(I_{\mathrm{D}} / I_{\mathrm{G}}\right)_{\mathrm{G}^{\prime} \text { ene }} \approx$ $\left(I_{\mathrm{D}} / I_{\mathrm{G}}\right)_{\mathrm{G}^{\prime} \text { ite }}+k /\langle L\rangle$, where $\left(I_{\mathrm{D}} / I_{\mathrm{G}}\right)_{\mathrm{G}^{\prime} \text { ite }}$ is the ratio measured on the starting graphite, $\langle L\rangle$ is the mean nanosheet length, $k$ is a parameter which depends on the Raman scattering process, the properties of the graphene itself and the nanosheet shape distribution. We have assumed that the starting natural flake parent graphites used have an in-plane lateral size much larger than the laser spot due to the large crystallite sizes (see Methods). Thus the D-band is dominated by contributions from basal plane defects of the parent graphite material which also remain in the exfoliated graphene. In Fig. 3C therefore we plot the $I_{\mathrm{D}} / I_{\mathrm{G}}$ ratio for graphene with the $I_{\mathrm{D}} / I_{\mathrm{G}}$ for the parent graphite subtracted: $\left(I_{\mathrm{D}} / I_{\mathrm{G}}\right)_{\mathrm{G}^{\prime} \text { ene }}-\left(I_{\mathrm{D}} / I_{\mathrm{G}}\right)_{\mathrm{G}^{\prime} \text { ite }}$. This plot makes it clear that there is a well-defined correlation between this value and the inverse of the nanosheet length. This correlation holds for graphene produced from the different parent graphite sources, where the relevant value of $I_{\mathrm{D}} / I_{\mathrm{G}}$ for each graphite is used and appears reasonably robust towards different exfoliation methods or stabilizers used in the LPE. It also holds for the more polydisperse stock-like dispersion. An empirical fit gives the average nanosheet length as:

$$
\langle L\rangle=\frac{0.094}{\left(I_{\mathrm{D}} / I_{\mathrm{G}}\right)_{\mathrm{G}^{\prime} \text { ene }}-\left(I_{\mathrm{D}} / I_{\mathrm{G}}\right)_{\mathrm{G}^{\prime} \text { ite }}}
$$

where $L$ is in $\mu \mathrm{m}$. From the scatter in the data used to generate this metric we estimate the relative error in the extracted values of $\langle L\rangle$ to be $\sim 20 \%$.

In contrast to measurements conducted on graphite nanocrystallites produced by heat treatment of diamond-like amor- phous carbon, ${ }^{49,63,64}$ we observe a significant D-band of 0.2 even in the case of the samples with mean nanosheet length as large as $800 \mathrm{~nm}$. In the case of the nanocrystallites, a significant D-band only evolved for nanosheets with crystallite sizes of $<100 \mathrm{~nm} .{ }^{64}$ In turn, measurements on ion-bombarded graphene $^{65}$ or $\mathrm{HOPG}^{66}$ show that the distance between basal plane defects should be $<25 \mathrm{~nm}$ to result in an $I_{\mathrm{D}} / I_{\mathrm{G}}$ ratio of $\sim 0.2$ (due to the different nature of basal plane/vacancy defects compared to edge defects). This may suggest that a significant portion of basal plane defects contribute to the measured D-band. While we cannot rule out that basal plane defects are present in the parent graphite (as suggested by $I_{\mathrm{D}} / I_{\mathrm{G}}$ ratios of up to 0.08 in the case of Sigma graphite with a typical crystallite size of $\sim 100 \mu \mathrm{m}$ ), we have subtracted this contribution from the plot in Fig. 3C. We therefore do not expect them to have a dramatic impact on the established metric. Another indicator that some basal plane defects are present is obtained from an analysis of the $I_{\mathrm{D}} / I_{\mathrm{D}^{\prime}}$ as suggested by Eckmann et al. ${ }^{67}$ (see ESI Fig. S25†). However, as previously analyzed in detail, ${ }^{13}$ it is unlikely that shear exfoliation introduces basal plane defects. This is in contrast to extended sonication, where introduction of basal plane defects has recently been reported. ${ }^{60}$ In addition, if the observed $I_{\mathrm{D}} / I_{\mathrm{G}}$ was due to basal plane defects, it would not be expected to scale linearly with the inverse nanosheet length. We therefore believe that the observed $I_{\mathrm{D}} / I_{\mathrm{G}}$ ratio is indeed related to edges and a result of the measurement on the films of restacked graphene. As outlined above, we sample roughly 25000 nanosheets per measurement and will therefore always detect nanosheet edges giving rise to most of the defect-induced scattering.

We also note that the constant $k(k=0.094$ here $)$ depends on the shape of the nanosheets (section S3.3†). We suggest that therefore, the data point from bath sonication marked by the cyan square in Fig. 3C and D is slightly offset from the expected fit line because nanosheets produced from different exfoliation techniques can have different aspect ratios, degrees of folding, etc. In addition, a significant deviation is observed when the spectrum is acquired on a liquid dispersion (see ESI Fig. $\mathrm{S} 7 \dagger$ ), probably due to orientation effects, while the metric seems to be less robust as nanosheets get small. This could also be due to different aspect ratios as nanosheets get very small $(<100 \mathrm{~nm})$. With these combined uncertainties in mind, it is therefore important to look for an alternative, more robust length metric as described below.

As previously observed by Cançado et al. in the case of nanographites, changes in crystallite size (or nanosheet size) are also reflected in peak broadening of the Raman peaks. ${ }^{49,64}$ Careful examination of Fig. 3A shows that there is indeed a clear discernible change in the width of the G-band with nanosheet size. We have plotted the FWHM of the G-band, $\Gamma_{\mathrm{G}}$, as a function of lateral size in Fig. 3E finding a well-defined correlation. The average lateral size can therefore be obtained from eqn (5):

$$
\langle L\rangle=50 \times \mathrm{e}^{-0.21 \times \Gamma_{\mathrm{G}}}
$$


where $\Gamma_{\mathrm{G}}$ is in $\mathrm{cm}^{-1}$. It is apparent that this offers an alternative metric for measurement of nanosheet size with all data from size-selected standard samples using different graphite sources, stock-like dispersion and samples obtained from different exfoliation methods and stabilizers collapsing on the same curve. The measurement of the liquid dispersion also yielded a similar result compared to the filtered film or dried droplet (Fig. S7†) suggesting that eqn (5) can also be applied in the case of liquid dispersion. The metric is therefore more robust than $I_{\mathrm{D}} / I_{\mathrm{G}}$ with respect to samples under analysis. In addition, it can be seen to be a more useful metric than $I_{\mathrm{D}} / I_{\mathrm{G}}$, as we can assume the G-width to be constant as long as the parent graphites are of reasonable structural quality. Hence, there is no need to take into account the defect content of the parent graphite. However, we have found the width metric to be slightly less robust against changes in measurement conditions, such as laser intensity and choice of grating (see ESI Fig. S20-S24†). From the scatter in the data used to generate this metric we estimate the relative error in the extracted values of $\langle L\rangle$ to be $\sim 20 \%$ which is similar to the $I_{\mathrm{D}} / I_{\mathrm{G}}$ metric.

As indicated above, Cançado et al. measured the dependence of $\Gamma_{\mathrm{G}}$ crystallite size for a range of different nano-graphite samples. ${ }^{49,64}$ They saw a similar size dependence, with peak widths close to those observed here, but for much smaller sizes (e.g. $\Gamma_{\mathrm{G}} \approx 27 \mathrm{~cm}^{-1}$ for $L \approx 35 \mathrm{~nm}$ ). While they quoted a size dependence of the form; $\Gamma_{\mathrm{G}}=A+B /\langle L\rangle$, replotting their data shows it to also fit well to an exponential expression like that shown in eqn (5), except with different values for the constants. While the similarities are encouraging, the reasons for these differences are not understood. We would like to note that - in contrast to the work of Cancado et al., our measurements are performed on restacked films of LPE few-layered graphene. Effects of tilting individual sheets or indeed probing an ensemble of restacked sheets can currently not be theoretically accounted for and are beyond the scope of this manuscript. Similar effects are expected for the thickness metrics outlined below. As such, our approaches to quantify spectral changes are empirically designed for LPE graphene.

Following the above discussion, we now propose a simple metric to describe the changes to the shape of the 2D-band as a function of nanosheet thickness. Even though measurements were conducted on restacked films of formerly exfoliated few-layer graphene, we observe systematic changes in the 2D-band with nanosheet thickness. This is because the graphene is not restacked with atomic registry, but is rotationally random. This scenario is very similar to that found in turbostatic graphite, where individual graphene monolayers are also randomly restacked. In turbostatic graphite, this random restacking results in $2 \mathrm{D}$ lineshapes that are similar to graphene, albeit broadened due to the relaxation of the double resonant Raman selection rules. ${ }^{46}$ That rotational angles have a dramatic impact on the 2D Raman mode has also been shown in the case of bilayer graphene. ${ }^{68}$ The fact that these restacked films resemble turbostatic graphite (albeit with varying layer numbers of the individual platelets) is further confirmed by an analysis of the 2D-band linewidth as presented in the ESI (Fig. S28†). We also note that residual surfactant/solvent that is adsorbed to the nanosheets may in fact further help to shield adjacent few-layer nanosheets from interacting. This is supported by the observation that we only observe minor changes in the 2D-band when we compare Raman spectra acquired on a filtered film and from a liquid dispersion (see ESI Fig. S7 $\dagger$ ) suggesting that the information on mean layer number can still be extracted from the shape of the $2 \mathrm{D}$ band in an ensemble of restacked nanosheets.

To establish a quantitative metric, we aim to keep the analysis as simple to apply as we can, avoiding fitting if possible. This is also because it would be extremely challenging to fit the 2D-band correctly, as we probe ensembles containing nanosheets of different thickness distributions. As evident from Fig. 3B, the simplest possible metric to account for the changes in the 2D-band for LPE graphene of different thickness is probably the intensity itself. We propose the intensity ratio (i.e. amplitudes) of the maximum of the $2 \mathrm{D}$-band to the G-band maximum (metric $M_{1}$ ), $M_{1}=I_{2 \mathrm{D}} / I_{\mathrm{G}}$, as potential metric for the determination of the number of layers. We have calculated this for all the samples studied here, finding a welldefined relationship with $N$, for $N<10$ layers (Fig. 3D). Empirical fitting allows us to propose a very simple metric to estimate $\langle N\rangle$ from the Raman spectrum of a restacked film:

$$
\langle N\rangle=1.04 M_{1}^{-2.32}
$$

From the scatter in the data used to generate this metric we estimate the relative error in the extracted values of $\langle N\rangle$ to be $\sim 25 \%$.

The great advantage of this metric is that it is very simple and quickly determined. It holds surprisingly well across almost all samples including the stock. However, we believe it is important to point out that the sample produced from exfoliation in a kitchen blender with fairy liquid as stabilizer (cyan square in Fig. 3E and F) is a clear outlier. The household detergent contains various surfactants, but also aromatic molecules that can adsorb rather strongly on the graphene. It is therefore possible that this metric is rather sensitive to doping effects. ${ }^{60-62}$ Despite this, we note that NMP and SC gave a similar $I_{2 \mathrm{D}} / I_{\mathrm{G}}$ versus $\langle N\rangle$ relationship suggesting that similar to absorbance and extinction spectra - the chemical environment does not have a dramatic impact on the spectral profile in the case of few-layer graphene. In addition, we note that the metric predicts that a monolayer should have a $2 \mathrm{D} / \mathrm{G}$ intensity ratio of $M_{1} \sim 1$. This is clearly not the case as monolayers usually display considerably larger ratios. ${ }^{54,69}$ This again shows that the Raman measurement on ensemble films of LPE graphene is somewhat different from measurements on individual sheets in terms of a quantification.

Recently, we suggested a simple ratio of intensities at fixed wavenumbers, normalized to the same ratio obtained from the parent graphite, as a method to describe changes in the shape of the 2D peak with nanosheet thickness. ${ }^{13}$ The positions we selected are the maximum and shoulder of the $2 \mathrm{D}$-band in the 
graphite spectrum. As the position of the shoulder is not precisely defined, we approximate this to be $30 \mathrm{~cm}^{-1}$ below the position of the maximum intensity. For our sample, these positions are $2720 \mathrm{~cm}^{-1}$ and $2690 \mathrm{~cm}^{-1}$, respectively. The calculated ratio for the graphene is then normalized to the ratio calculated for the parent graphite. The metric is therefore calculated as

$$
M_{2}=\frac{\left[I_{\omega_{1}} / I_{\omega_{2}}\right]_{\mathrm{G}^{\prime} \text { ene }}}{\left[I_{\omega_{1}} / I_{\omega_{2}}\right]_{\mathrm{G}^{\prime} \text { ite }}}
$$

where $\omega_{1}=2720 \mathrm{~cm}^{-1}$ (position of graphite maximum) and $\omega_{2}$ $=\left(\omega_{1}-30\right)=2690 \mathrm{~cm}^{-1}$. We originally developed this metric using literature data on micromechanically cleaved graphene nanosheets, where the layer number of the individual nanosheets, $N_{\mathrm{G}}$, is precisely known. ${ }^{13}$ In this well-defined case, we found a well-defined relationship between $M_{2}$ and layer number: $N_{\mathrm{G}}=10^{0.84 M_{2}+0.45 M_{2}{ }^{2}}$.

We have calculated $M_{2}$ from the Raman data collected in this study and plotted it versus $\langle N\rangle$ in Fig. 3F. We find a welldefined relationship, however one that is quantitatively different to that predicted by the empirical equation (above) generated from mechanically cleaved graphene. We suggest that this is due to environmental differences between cleaved graphene and the liquid exfoliated graphene under study here (restacking, residual solvent or surfactant, strain, orientation, etc.) as we have observed throughout our study. We have found an empirical fit to the data in Fig. $3 \mathrm{~F}$ which described all data very well (dashed line). This leads to the metric for $\langle N\rangle$ :

$$
\langle N\rangle=0.83 \times \mathrm{e}^{3.6 M_{2}}
$$

From the scatter in the data used to generate this metric we estimate the relative error in the extracted values of $\langle N\rangle$ to be $\sim 18 \%$.

However, this metric has the disadvantage that it is very sensitive to shifts in the band positions in the exfoliated graphene relative to the starting graphite so that the spectrometer needs to be carefully calibrated prior to the measurement. In addition, local heating when acquiring the spectra at high laser power (>2 $\mathrm{mW}$ ) reduces the $M_{2}$ metric values (Fig. S23 $\dagger$ ) making the graphene look thinner. However, unlike the $I_{2 \mathrm{D}} / I_{\mathrm{G}}$ metric, $M_{2}$ is quite robust towards different samples, as all data, even the graphene produced from exfoliation in a kitchen blender with fairy liquid as stabilizer falls on the same master curve. This suggests even doping effects to be negligible when an intensity ratio of the 2D-band itself is analyzed. There are a number of other alternatives to quantify spectral changes of the $2 \mathrm{D}$-band such as full width at half maximum or the intensity ratio of the 2D-band to the G-band at fixed spectral positions. Details of some of these are shown and discussed in the ESI Fig. S26-S31. $\dagger$

Finally, we note that we have established quantitative relationships between spectroscopic data and number mean values of length and thickness. Technically, spectroscopic techniques are integral and probe volume/mass rather than number population. However, as described in detail in the ESI, section $4, \dagger$ we observe a well-defined correlation between number mean values and volume fraction weighted mean values of both thickness and length (Fig. S33†). Therefore, number mean values can indeed be extracted from a spectroscopic analysis (albeit with a different quantification compared to volume fraction weighted mean, Fig. S34 and S35†).

\section{Conclusions}

In conclusion, we have produced a series of water-surfactant based dispersions of graphene with varying lateral size and thickness distributions obtained by liquid cascade centrifugation. Both lateral size and thickness have been quantified by AFM and TEM. By measuring the extinction and absorbance spectra of these dispersions, we have identified systematic changes of the spectra as a function of nanosheet size. By comparing these changes to the nanosheet size measured by microscopy, we have presented quantitative metrics for the nanosheet thickness. For example, the position of the $\pi-\pi^{*}$ absorbance changes as a function of thickness, as confirmed by theory. In addition, spectral changes can be expressed as peak intensity ratios such as intensity at the peak to the high wavelength plateau. This also provides a robust metric for graphene thickness from both absorbance and extinction spectra.

We have also used Raman spectroscopy on filtered films of the dispersions to provide quantitative metrics for graphene nanosheet lateral size and thickness. While Raman is currently widely used to provide qualitative measures of nanosheet size, we have shown that quantitative metrics can be obtained from the Raman spectra of restacked films of LPE nanosheets. The lateral size can be quantified by either the intensity ratio of the D-band to G-band, or by the width of the G-band. Similarly, the average number of layers can be quantified either by the intensity ratio of the $2 \mathrm{D}$-to G-band or of two positions of the 2D-band. To our knowledge, this is the first description of how layer number and nanosheet length of liquid exfoliated fewlayer graphene can be quantitatively extracted from Raman spectra of filtered films.

However, we note that although we have found the metrics to hold for different common graphite sources exfoliated by different methods and stabilized in different environments, we cannot be absolutely certain that they apply perfectly to all of the many types of samples available. Thus we recommend that use of these metrics is combined with the occasional confirmation using statistical TEM or AFM. In this way, a huge amount of time and effort can be saved while still retaining complete confidence in the results.

The metrics we have presented here provide the low cost and high throughput characterization techniques that are needed for the commercial exploitation of graphene. By removing the need for costly and time-consuming microscopy-based techniques, the process of production optimization can also be accelerated. This will allow the development of graphene products that are better tailored to specific applications. By using measurement equipment that is more widely accessible 
with lower costs, these metrics also make the characterization of graphene easier for end-users of graphene. This is likely to encourage further innovation and exploration of novel applications of graphene. In this way, the remarkable properties of graphene can be fully exploited in commercial applications.

\section{Methods}

\section{Materials}

Sodium cholate (SC) was purchased from Sigma Aldrich (C1254), N-methyl-2-pyrrolidone (NMP) from VWR (Emplura® grade) and used as received. Different natural flake graphites were obtained from (i) Sigma Aldrich (grade 332461, flake size $\sim 100 \mu \mathrm{m}$ ), (ii) Asbury Carbons (grade 3763, flake size $460 \mu \mathrm{m}$ ), (iii) Qingdao Henglide Graphite Co Ltd (natural flake graphite, +32 mesh, flake size $500 \mu \mathrm{m}$ ) and (iv) Imerys Graphite and Carbon (Timrex $20 \times 50$, flake size $300 \mu \mathrm{m}$ ). To acquire the Raman spectra of the parent graphites, edge regions were avoided and the laser was focused on basal plane areas with no visible terraces in the optical micrographs. 5-10 measurements were averaged.

\section{Preparation of the standard dispersions}

Dispersions of graphene in aqueous surfactant solutions were prepared by shear mixing using a Silverson L5M rotor-stator mixer, as detailed previously. ${ }^{14}$ A solution of sodium cholate in de-ionised water $\left(1 \mathrm{~g} \mathrm{~L}^{-1}\right)$ was prepared and then added to natural flake graphite (Sigma-Aldrich, 332461, or Asbury, grade 3763 ) at $100 \mathrm{~g} \mathrm{~L}^{-1}$ with a typical volume of $1 \mathrm{~L}$. Graphene was exfoliated using a $32 \mathrm{~mm}$ rotor at $4500 \mathrm{rpm}$ for $60 \mathrm{~min}$. In order to remove some low mass impurities (which we found to partly destabilize the dispersion and thus inhibit centrifugation-based size selection), the resulting dispersion was left to settle overnight, and the supernatant discarded. Fresh surfactant solution was then added and the exfoliation repeated as before. The dispersion was left to settle for several hours before subjecting the supernatant to the centrifugation-based size selection.

\section{Size selection of the standard dispersions}

To select nanosheets by size, we used controlled centrifugation with sequentially increasing rotation speeds as previously reported. ${ }^{14,27}$ An initial centrifugation at $1.5 \mathrm{krpm}(240 g, 2 \mathrm{~h})$ was performed to remove unexfoliated material. The supernatant was subjected to further centrifugation at $2 \mathrm{krpm}(425 \mathrm{~g}$, $2 \mathrm{~h}$ ). The sediment was collected in fresh water-SC (SC concentration $0.1 \mathrm{~g} \mathrm{~L}^{-1}$ to facilitate imaging), while the supernatant was subjected to further centrifugation at $2.5 \mathrm{krpm}(665 g, 2 \mathrm{~h})$. Again, the sediment was collected and the supernatant centrifuged at higher rpm. This procedure was repeated for $3 \mathrm{krpm}$ (958g, 2 h), 5 krpm (2660g, 2 h), $10 \mathrm{krpm}(10170 g, 2 \mathrm{~h})$ and $18 \mathrm{krpm}(32600 \mathrm{~g}, 2 \mathrm{~h})$ to yield samples with decreasing sizes in the respective sediments. Compared with a method based on taking the supernatant from a single step centrifugation, this produces dispersions with lower polydispersity, as both smaller and larger nanosheets are removed from a given sizeselected dispersion. This greatly facilitates microscopic characterization required to accurately determine length and thickness. A Hettich Mikro 220R centrifuge equipped with a fixedangle rotor 1016 and sample aliquots of $10 \mathrm{~mL}$ was used for rotations up to $5 \mathrm{krpm}$. For the high speed centrifugations, $1.5 \mathrm{~mL}$ vials and a rotor 1195-A was used. In the case of the analysis of the stock-like dispersion, the sample of Asbury graphite exfoliated in SC was centrifuged at $1 \mathrm{krpm}(104 \mathrm{~g}, 2 \mathrm{~h})$ and the sediment discarded. We note that the removal of nonexfoliated graphite is required for the metrics to be applicable.

\section{Special dispersions}

The dispersion of Sigma Aldrich graphite exfoliated in a kitchen blender with fairy liquid as stabilizer was produced as follows: Flake graphite was purchased from Sigma Aldrich (Product no. 332461) and used as supplied. The surfactant used was Fairy washing-up liquid (Fairy Liquid, FL), a common household dishwashing liquid, with a composition of $15-30 \%$ anionic surfactants, 5-15\% non-ionic surfactants (specified by the manufacturer, Proctor \& Gamble). A Kenwood kitchen blender (model BL 370) was used. $2.5 \mathrm{~g} \mathrm{~L}^{-1}$ of FL was dissolved in deionized water and graphite $\left(20 \mathrm{~g} \mathrm{~L}^{-1}\right)$ was added to the tapered blender jug and FL/water solution poured in on top. The blender was operated at full speed for $60 \mathrm{~min}$. Such blenders are not designed for continuous operation at high speeds for long times due to excess heating. To counter this, the mixer was turned off for one minute after every minute of mixing ( $1 \mathrm{~min}$ on/1 min off duty cycle). The jug was kept in an ice bath during the off cycles. The resultant dispersion was left to settle overnight prior to centrifugation. The FLG sedimenting between $1.5 \mathrm{krpm}(240 \mathrm{~g}$ ) and $7.5 \mathrm{krpm}$ (5400g) was collected in deionized water and subjected to analysis.

To produce the graphene dispersion exfoliated in a sonic bath in aqueous sodium cholate, the parent graphite (Timrex $20 \times 50$, Imerys Graphite and Carbon, $100 \mathrm{~g} \mathrm{~L}^{-1}$ ) was immersed in aqueous sodium cholate $\left(2 \mathrm{~g} \mathrm{~L}^{-1}\right)$ and sonicated in $4 \times$ $50 \mathrm{ml}$ vials in a water cooled sonic bath for $410 \mathrm{~min}$. The resultant dispersion was left to settle overnight prior to centrifugation. The FLG sedimenting between $3 \mathrm{krpm}$ (958g) and $5 \mathrm{krpm}(2660 \mathrm{~g})$ was collected in water-SC and subjected to analysis.

The samples of Qingdao graphite (Qingdao Henglide Graphite Co Ltd, natural flake graphite, +32 mesh) exfoliated using a rotor-stator mixer in SC and $N$-methyl-2-pyrrolidone (NMP) were produced in analogy to the standard samples described above. NMP was purchased from VWR (Emplura® grade) and used as received. The dispersions were left to settle overnight prior to centrifugation. The FLG sedimenting between $3 \mathrm{krpm}(958 g)$ and $5 \mathrm{krpm}$ (2660g) was collected in water-SC or fresh NMP, respectively and subjected to analysis.

\section{Characterization and equipment}

Low-resolution bright field transmission electron microscopy imaging was performed using a JEOL 2100, operated at $200 \mathrm{kV}$. Holey carbon grids (400 mesh) were purchased from Agar 
Scientific and prepared by diluting dispersion to a low concentration and drop casting onto a grid placed on a filter membrane to wick away excess solvent. Statistical analysis was performed of the nanosheet dimensions by measuring the longest axis of the nanosheet and assigning it "length, $L$ ".

Atomic force microscopy (AFM) was carried out on a Veeco Nanoscope-IIIa (Digital Instruments) system equipped with an E-head (13 $\mu \mathrm{m}$ scanner) in tapping mode after depositing a drop of the dilute dispersions $(10 \mu \mathrm{L}$, optical density at high wavelength $\sim 0.1)$ on a pre-heated $\left(120{ }^{\circ} \mathrm{C}\right) \mathrm{Si} / \mathrm{SiO}_{2}$ wafer $\left(0.5 \times 0.5 \mathrm{~cm}^{2}\right)$ with an oxide layer of $300 \mathrm{~nm}$. The wafers were washed with $\sim 5 \mathrm{~mL}$ of water and isopropanol. Typical image sizes were $2-5 \mu \mathrm{m}$ at scan rates of $0.5-0.8 \mathrm{~Hz}$. The mean number of layers from the measured apparent thickness of the nanosheets was obtained using the previously developed step height analysis. ${ }^{13}$

Optical extinction and absorbance was measured on a Perkin Elmer 650 spectrometer in quartz cuvettes with a path length of $0.4 \mathrm{~cm}$. To distinguish between contributions from scattering and absorbance to the extinction spectra, dispersions were measured in an integrating sphere using a homebuilt sample holder to place the cuvette in the center of the sphere (NB cuvettes need to be transparent to all sides). The absorbance spectrum is obtained from the measurement inside the sphere. A second measurement on each dispersion was performed outside the sphere in the standard configuration to obtain the extinction spectrum. This allows calculation of the scattering spectrum (extinction minus absorbance). Extinction, absorbance and scattering coefficients were determined by filtration (alumina membranes, $20 \mathrm{~nm}$ pore size) and weighing. Most of the surfactant was removed by washing with $400 \mathrm{~mL}$ of deionized water in the filtration step. TGA was used to measure the quantity of residual surfactant (Fig. S11†) to correct the measured mass and hence extinction coefficients. Measurements were carried out using a Perkin Elmer Pyris 1 TGA, using a heating rate of $10^{\circ} \mathrm{C} \mathrm{min}^{-1}$ in dry air flow.

Raman spectroscopy was performed on filtered films of the dispersions (alumina membranes, pore size $20 \mathrm{~nm}$ ) using a Horiba Jobin Yvon LabRAM HR800 with $532 \mathrm{~nm}$ excitation laser in air under ambient conditions. The Raman emission was collected by $100 \times$ objective lens $($ N.A. $=0.8$, spot size $\sim$ $1 \mu \mathrm{m})$ and dispersed by $600 \mathrm{~g} \mathrm{~mm}^{-1}$ unless otherwise noted. To avoid sample heating we carried out all Raman experiments at $10 \%$ of maximum laser power $(<2 \mathrm{~mW})$ unless otherwise noted. The impact of heating and the choice of the grating on the Raman spectra is discussed in the ESI section 3.1 and 3.2. $\dagger$ A mapping over a $20 \times 20 \mu^{2}$ sample area was performed in each case in $2 \mu \mathrm{m}$ steps. The spectra displayed are the baseline-corrected average of 120 individual spectra. No impact on the metric values from averaging was observed as discussed in the ESI section 3.6. $\dagger$

\section{Numerical methods}

To study the thickness dependence of the optical properties we performed numerical calculations of the ABA-stacked (and ABC stacked, see ESI $\dagger$ ) multilayer graphene by using the tight- binding propagation method (TBPM). ${ }^{35,36}$ The real part of the optical conductivity is calculated via the Kubo's formula as

$\sigma(\omega)=\lim _{\varepsilon \rightarrow+0} \frac{1-e^{-\beta \hbar \omega}}{\hbar \omega \Omega} \int_{0}^{\infty} d t e^{i(\omega+i \varepsilon) \tau} 2 i \operatorname{Im}\langle\phi|J[1-f(H)] J(\tau) f(H)| \phi\rangle$,

where $\Omega$ is the sample area, $\beta=1 / k_{\mathrm{B}} T$ is the inverse temperature, $H$ is the tight-binding Hamiltonian, $f(H)=[\exp [\beta(H-\mu)]$ $+1]^{-1}$ is the Fermi-Dirac distribution operator, and $J(\tau)=$ $e^{i H \tau / \hbar} J e^{-i H \tau / \hbar}$ is the current operator in the Heisenberg picture. The state $|\phi\rangle$ is a normalized random state which covers all the eigenstates in the whole spectrum. ${ }^{35}$ The time evolution operator and Fermi-Dirac distribution operator are represented as the Chebyshev polynomial expansions. Here we omit the Drude weight since we are interested only in light adsorption at finite $\omega$.

In order to phenomenologically implement the red shift of the absorbance spectrum due to the excitonic effect, the intralayer hopping energy between two nearest neighbors is set to be $t=2.3 \mathrm{eV}$, the value which leads to the match of the calculated $\pi$-excitonic peak at $2 t$ and the experimental observed peak at $4.6 \mathrm{eV}$ for single-layer graphene. The interlayer hopping parameters between the atomic sites in two nearest layers are set to be $t_{1}=0.13 t$ and $t_{3}=0.1 t .^{70,71}$

The optical transmittance through a graphene thin film is calculated from the optical conductivity as ${ }^{72}$

$$
T(\omega)=\left[1+\frac{2 \pi}{c} \sigma(\omega)\right]^{-2},
$$

and the optical absorbance is found from $A(\omega)=-\log T(\omega)$.

The method implemented here has the advantage that the CPU time and the memory costs are both linear dependent on the sample size, and the presented numerical results are obtained from samples consisting of $\sim 10^{9}$ atomic sites.

\section{Acknowledgements}

The research leading to these results has received funding from the European Union Seventh Framework Programme under grant agreement no 604391 Graphene Flagship. In addition, we acknowledge Science Foundation Ireland (11/PI/ 1087), the European Research Council (SEMANTICS and POC grant UP2DM) and Thomas Swan \& Co. Ltd for financial support. CB acknowledges the German research foundation DFG (BA 4856/1-1). We thank Asbury Carbons and Imerys Graphite and Carbon for suppling parent graphite materials free of charge.

\section{References}

1 A. K. Geim, Science, 2009, 324, 1530-1534.

2 F. Bonaccorso, L. Colombo, G. H. Yu, M. Stoller, V. Tozzini, A. C. Ferrari, R. S. Ruoff and V. Pellegrini, Science, 2015, $347,10$. 
3 K. S. Novoselov, V. I. Fal'ko, L. Colombo, P. R. Gellert, M. G. Schwab and K. Kim, Nature, 2012, 490, 192-200.

4 K. S. Novoselov, D. Jiang, F. Schedin, T. J. Booth, V. V. Khotkevich, S. V. Morozov and A. K. Geim, Proc. Natl. Acad. Sci. U. S. A., 2005, 102, 10451-10453.

5 X. Li, W. Cai, J. An, S. Kim, J. Nah, D. Yang, R. Piner, A. Velamakanni, I. Jung, E. Tutuc, S. K. Banerjee, L. Colombo and R. S. Ruoff, Science, 2009, 324, 1312-1314.

6 V. Nicolosi, M. Chhowalla, M. G. Kanatzidis, M. S. Strano and J. N. Coleman, Science, 2013, 340, 1226419.

7 R. Ruoff, Nat. Nanotechnol., 2008, 3, 10-11.

8 Y. Hernandez, V. Nicolosi, M. Lotya, F. M. Blighe, Z. Sun, S. De, I. T. McGovern, B. Holland, M. Byrne, Y. K. Gun'Ko, J. J. Boland, P. Niraj, G. Duesberg, S. Krishnamurthy, R. Goodhue, J. Hutchison, V. Scardaci, A. C. Ferrari and J. N. Coleman, Nat. Nanotechnol., 2008, 3, 563-568.

9 A. Ciesielski and P. Samori, Chem. Soc. Rev., 2014, 43, 381398.

10 Y. Hernandez, M. Lotya, D. Rickard, S. D. Bergin and J. N. Coleman, Langmuir, 2010, 26, 3208-3213.

11 J. N. Coleman, M. Lotya, A. O’Neill, S. D. Bergin, P. J. King, U. Khan, K. Young, A. Gaucher, S. De, R. J. Smith, I. V. Shvets, S. K. Arora, G. Stanton, H.-Y. Kim, K. Lee, G. T. Kim, G. S. Duesberg, T. Hallam, J. J. Boland, J. J. Wang, J. F. Donegan, J. C. Grunlan, G. Moriarty, A. Shmeliov, R. J. Nicholls, J. M. Perkins, E. M. Grieveson, K. Theuwissen, D. W. McComb, P. D. Nellist and V. Nicolosi, Science, 2011, 331, 568-571.

12 R. J. Smith, P. J. King, M. Lotya, C. Wirtz, U. Khan, S. De, A. O’Neill, G. S. Duesberg, J. C. Grunlan, G. Moriarty, J. Chen, J. Wang, A. I. Minett, V. Nicolosi and J. N. Coleman, Adv. Mater., 2011, 23, 3944-3948.

13 K. R. Paton, E. Varrla, C. Backes, R. J. Smith, U. Khan, A. O'Neill, C. Boland, M. Lotya, O. M. Istrate, P. King, T. Higgins, S. Barwich, P. May, P. Puczkarski, I. Ahmed, M. Moebius, H. Pettersson, E. Long, J. Coelho, S. E. O’Brien, E. K. McGuire, B. M. Sanchez, G. S. Duesberg, N. McEvoy, T. J. Pennycook, C. Downing, A. Crossley, V. Nicolosi and J. N. Coleman, Nat. Mater., 2014, 13, 624-630.

14 E. Varrla, K. R. Paton, C. Backes, A. Harvey, R. J. Smith, J. McCauley and J. N. Coleman, Nanoscale, 2014, 6, 1181011819.

15 L. Liu, Z. Shen, M. Yi, X. Zhang and S. Ma, RSC Adv., 2014, 4, 36464-36470.

16 P. May, U. Khan, A. O'Neill and J. N. Coleman, J. Mater. Chem., 2012, 22, 1278-1282.

17 L. Liu, Z. G. Shen, S. S. Liang, M. Yi, X. J. Zhang and S. L. Ma, J. Mater. Sci., 2014, 49, 321-328.

18 G. P. Keeley, A. O’Neill, N. McEvoy, N. Peltekis, J. N. Coleman and G. S. Duesberg, J. Mater. Chem., 2010, 20, 7864-7869.

19 S. P. Qi, B. Zhao, H. Q. Tang and X. Q. Jiang, Electrochim. Acta, 2015, 161, 395-402.

20 C. S. Boland, U. Khan, C. Backes, A. O’Neill, J. McCauley, S. Duane, R. Shanker, Y. Liu, I. Jurewicz, A. B. Dalton and J. N. Coleman, ACS Nano, 2014, 8, 8819-8830.
21 S. Mutyala and J. Mathiyarasu, Sens. Actuator, B, 2015, 210, 692-699.

22 D. J. Finn, M. Lotya, G. Cunningham, R. J. Smith, D. McCloskey, J. F. Donegan and J. N. Coleman, J. Mater. Chem. C, 2014, 2, 925-932.

23 F. Torrisi, T. Hasan, W. Wu, Z. Sun, A. Lombardo, T. S. Kulmala, G.-W. Hsieh, S. Jung, F. Bonaccorso, P. J. Paul, D. Chu and A. C. Ferrari, ACS Nano, 2012, 6, 2992-3006.

24 L. Zhang, J. T. Fan, J. H. Wang, J. M. Hu, M. Lotya, G. Z. Wang, R. H. Li, L. Zhang, W. J. Blau, J. N. Coleman, J. Wang and Y. Feng, Laser Phys. Lett., 2012, 9, 888-892.

25 T. Hasan, F. Torrisi, Z. Sun, D. Popa, V. Nicolosi, G. Privitera, F. Bonaccorso and A. C. Ferrari, Phys. Status Solidi B, 2010, 247, 2953-2957.

26 L. Kavan, J. H. Yum and M. Gratzel, ACS Nano, 2011, 5, 165-172.

27 U. Khan, A. O'Neill, H. Porwal, P. May, K. Nawaz and J. N. Coleman, Carbon, 2012, 50, 470-475.

28 A. A. Green and M. C. Hersam, Nano Lett., 2009, 9, 40314036.

29 C. Backes, R. J. Smith, N. McEvoy, N. C. Berner, D. McCloskey, H. C. Nerl, A. O’Neill, P. J. King, T. Higgins, D. Hanlon, N. Scheuschner, J. Maultzsch, L. Houben, G. S. Duesberg, J. F. Donegan, V. Nicolosi and J. N. Coleman, Nat. Commun., 2014, 5, 4576.

30 E. Varrla, C. Backes, K. R. Paton, A. Harvey, Z. Gholamvand, J. McCauley and J. N. Coleman, Chem. Mater., 2015, 27, 1129-1139.

31 A. Harvey, C. Backes, Z. Gholamvand, D. Hanlon, D. McAteer, H. C. Nerl, E. McGuire, A. Seral-Ascaso, Q. M. Ramasse, N. McEvoy, S. Winters, N. C. Berner, D. McCloskey, J. F. Donegan, G. S. Duesberg, V. Nicolosi and J. N. Coleman, Chem. Mater., 2015, 27, 3483-3493.

32 C. Backes, B. M. Szydłowska, A. Harvey, S. Yuan, V. VegaMayoral, B. R. Davies, P.-l. Zhao, D. Hanlon, E. J. G. Santos, M. I. Katsnelson, W. J. Blau, C. Gadermaie and J. N. Coleman, ACS Nano, 2016, 10(1), 1589-1601.

33 D. Hanlon, C. Backes, E. Doherty, C. S. Cucinotta, N. C. Berner, C. Boland, K. Lee, P. Lynch, Z. Gholamvand, A. Harvey, S. Zhang, K. Wang, G. Moynihan, A. Pokle, Q. M. Ramasse, N. McEvoy, W. J. Blau, J. Wang, G. Abellan, F. Hauke, A. Hirsch, S. Sanvito, D. D. O'Regan, G. S. Duesberg, V. Nicolosi and J. N. Coleman, Nat. Commun., 2015, 6, 8563.

34 D. Hanlon, C. Backes, T. M. Higgins, M. Hughes, A. O'Neill, P. King, N. McEvoy, G. S. Duesberg, B. Mendoza Sanchez, H. Pettersson, V. Nicolosi and J. N. Coleman, Chem. Mater., 2014, 26, 1751-1763.

35 S. Yuan, H. De Raedt and M. I. Katsnelson, Phys. Rev. B: Condens. Matter, 2010, 82, 115448.

36 S. Yuan, R. Roldán, H. De Raedt and M. I. Katsnelson, Phys. Rev. B: Condens. Matter, 2011, 84, 195418.

37 L. Yadgarov, C. L. Choi, A. Sedova, A. Cohen, R. Rosentsveig, O. Bar-Elli, D. Oron, H. Dai and R. Tenne, ACS Nano, 2014, 8, 3575-3583. 
38 R. R. Nair, P. Blake, A. N. Grigorenko, K. S. Novoselov, T. J. Booth, T. Stauber, N. M. R. Peres and A. K. Geim, Science, 2008, 320, 1308-1308.

39 A. O'Neill, U. Khan and J. N. Coleman, Chem. Mater., 2012, 24, 2414-2421.

40 M. Lotya, Y. Hernandez, P. J. King, R. J. Smith, V. Nicolosi, L. S. Karlsson, F. M. Blighe, S. De, Z. Wang, I. T. McGovern, G. S. Duesberg and J. N. Coleman, J. Am. Chem. Soc., 2009, 131, 3611-3620.

41 M. Lotya, P. J. King, U. Khan, S. De and J. N. Coleman, ACS Nano, 2010, 4, 3155-3162.

42 U. Khan, A. O’Neill, M. Lotya, S. De and J. N. Coleman, Small, 2010, 6, 864-871.

43 D. Ager, V. A. Vasantha, R. Crombez and J. Texter, ACS Nano, 2014, 8, 11191-11205.

44 K. R. Paton and J. N. Coleman, 2015, arXiv, submit/ 1404279.

45 A. C. Ferrari and D. M. Basko, Nat. Nanotechnol., 2013, 8, 235-246.

46 L. M. Malard, M. A. Pimenta, G. Dresselhaus and M. S. Dresselhaus, Phys. Rep., 2009, 473, 51-87.

47 I. Calizo, A. A. Balandin, W. Bao, F. Miao and C. N. Lau, Nano Lett., 2007, 7, 2645-2649.

48 F. Hof, S. Bosch, J. M. Englert, F. Hauke and A. Hirsch, Angew. Chem., Int. Ed., 2012, 51, 11727-11730.

49 L. G. Cançado, A. Jorio and M. A. Pimenta, Phys. Rev. B: Condens. Matter, 2007, 76, 064304.

50 F. Tuinstra and J. L. Koenig, J. Chem. Phys., 1970, 53, 11261130.

51 C. Casiraghi, A. Hartschuh, H. Qian, S. Piscanec, C. Georgi, A. Fasoli, K. S. Novoselov, D. M. Basko and A. C. Ferrari, Nano Lett., 2009, 9, 1433-1441.

52 A. C. Ferrari and J. Robertson, Phys. Rev. B: Condens. Matter, 2000, 61, 14095-14107.

53 A. C. Ferrari, J. C. Meyer, V. Scardaci, C. Casiraghi, M. Lazzeri, F. Mauri, S. Piscanec, D. Jiang, K. S. Novoselov, S. Roth and A. K. Geim, Phys. Rev. Lett., 2006, 97, 187401.

54 D. Yoon, H. Moon, H. Cheong, J. S. Choi, J. A. Choi and B. H. Park, J. Korean Phys. Soc., 2009, 55, 1299-1303.

55 A. Ciesielski, S. Haar, M. El Gemayel, H. Yang, J. Clough, G. Melinte, M. Gobbi, E. Orgiu, M. V. Nardi, G. Ligorio, V. Palermo, N. Koch, O. Ersen, C. Casiraghi and P. Samorì, Angew. Chem., Int. Ed., 2014, 53, 10355-10361.
56 S. Haar, A. Ciesielski, J. Clough, H. Yang, R. Mazzaro, F. Richard, S. Conti, N. Merstorf, M. Cecchini, V. Morandi, C. Casiraghi and P. Samorì, Small, 2015, 11, 1691-1702.

57 T. Nacken, C. Damm, H. Xing, A. Rüger and W. Peukert, Nano Res., 2015, 8, 1865-1881.

58 C. Damm, T. J. Nacken and W. Peukert, Carbon, 2015, 81, 284-294.

59 S. De, P. J. King, M. Lotya, A. O’Neill, E. M. Doherty, Y. Hernandez, G. S. Duesberg and J. N. Coleman, Small, 2010, 6, 458-464.

60 D. M. Basko, S. Piscanec and A. C. Ferrari, Phys. Rev. B: Condens. Matter, 2009, 80, 165413.

61 M. Bruna, A. K. Ott, M. Ijäs, D. Yoon, U. Sassi and A. C. Ferrari, ACS Nano, 2014, 8, 7432-7441.

62 G. Froehlicher and S. Berciaud, Phys. Rev. B: Condens. Matter, 2015, 91, 205413.

63 L. G. Cançado, K. Takai, T. Enoki, M. Endo, Y. A. Kim, H. Mizusaki, A. Jorio, L. N. Coelho, R. Magalhães-Paniago and M. A. Pimenta, Appl. Phys. Lett., 2006, 88, 163106.

64 V. Vega-Mayoral, C. Backes, D. Hanlon, U. Khan, Z. Gholamvand, M. O'Brien, G. S. Duesberg, C. Gadermaier and J. N. Coleman, Adv. Funct. Mater., 2015, DOI: 10.1002/ adfm.201503863, in press.

65 E. B. Secor and M. C. Hersam, J. Phys. Chem. Lett., 2015, 6, 620-626.

66 M. M. Lucchese, F. Stavale, E. H. M. Ferreira, C. Vilani, M. V. O. Moutinho, R. B. Capaz, C. A. Achete and A. Jorio, Carbon, 2010, 48, 1592-1597.

67 A. Eckmann, A. Felten, A. Mishchenko, L. Britnell, R. Krupke, K. S. Novoselov and C. Casiraghi, Nano Lett., 2012, 12, 3925-3930.

68 K. Kim, S. Coh, L. Z. Tan, W. Regan, J. M. Yuk, E. Chatterjee, M. F. Crommie, M. L. Cohen, S. G. Louie and A. Zettl, Phys. Rev. Lett., 2012, 108, 246103.

69 A. C. Ferrari, J. C. Meyer, V. Scardaci, C. Casiraghi, M. Lazzeri, F. Mauri, S. Piscanec, D. Jiang, K. S. Novoselov, S. Roth and A. K. Geim, Phys. Rev. Lett., 2006, 97, 187401.

70 A. H. Castro Neto, F. Guinea, N. M. R. Peres, K. S. Novoselov and A. K. Geim, Rev. Mod. Phys., 2009, 81, 109-162.

71 M. I. Katsnelson, Graphene- Carbon in Two Dimensions, Cambridge Univ. Press, Cambridge, 2012.

72 H. Min and A. H. MacDonald, Phys. Rev. Lett., 2009, 103, 067402 . 